\title{
Optimization of nanocavity field enhancement using two-dimensional plasmonic photonic crystals
}

\author{
TAO Xing \& DONG ZhenChao* \\ Hefei National Laboratory for Physical Sciences at the Microscale, University of Science and Technology of China, Hefei 230026, China
}

Received May 11, 2011; accepted July 1, 2011

\begin{abstract}
We have investigated the influence of $\mathrm{Ag}$ nanorod radius $(r)$ on the resonant modes of a two-dimensional plasmonic photonic crystal (PPC) with dipole sources embedded into the central vacancy area, using finite-difference time-domain methods. Both the localized surface plasmon (LSP) mode of individual Ag nanorods and the resonant cavity mode of PPC are found to vary as a function of $r$. The resonant cavity mode is strongly enhanced as $r$ is increased, while the LSP signal will eventually become no longer discernable in the Fourier spectrum of the time-evolved field. An optimized condition for the nanocavity field enhancement is found for a given PPC periodicity (e.g. $d=375 \mathrm{~nm}$ ) with the critical nanorod radius $r_{\mathrm{c}}=d / 3$. At this point the resonant cavity mode has the strongest field enhancement, best field confinement and largest $Q$-factor. We attribute this to competition between the blocking of cavity confined light to radiate out when the cavity resonant frequency falls inside the opened photonic stopband as $r$ reaches $r_{\mathrm{c}}$, and the transfer of cavity mode energy to inter-particle plasmons when $r$ is further increased.
\end{abstract}

plasmonic photonic crystal, finite-difference time-domain method, localized surface plasmon, Purcell factor

Citation: Tao X, Dong Z C. Optimization of nanocavity field enhancement using two-dimensional plasmonic photonic crystals. Chin Sci Bull, 2012, 57: 77-82, doi: $10.1007 / \mathrm{s} 11434-011-4832-1$

Light manipulation on the nanoscale is an important goal in nano-photonics [1]. An interesting material in this field is metallic nanostructures that support local surface plasmons (LSP). The highly confined electromagnetic field associated with LSP excitation can greatly enhance the efficiency of many optical processes [2], including surface-enhanced Raman scattering [3-5] and metal-enhanced fluorescence [6,7]. There are two main strategies to achieve high field enhancement: one is to use the "nanogap" effect through the coupling of two metallic nanostructures $[3,8]$, and the other is to use plasmonic photonic crystals (PPC) $[9,10]$. The surface plasmon polaritons (SPP) in a PPC structure can be partly trapped in a nanocavity as a cavity plasmon mode. Thus, they offer the potential to manipulate light in a confined space and to realize strong field enhancement at the nanoscale [11]. By introducing a defect into a PPC, a strong SPP resonant defect mode can be excited at the defect regime by Bragg reflection [12,13].

*Corresponding author (email: zcdong@ustc.edu.cn)
Recently, we demonstrated the generation of anomalous molecular electroluminescence, the so-called "forbidden light" by resonant plasmons that are highly confined inside a nanogap between metallic nanotip and metal substrate in a scanning tunneling microscope $[14,15]$. However, the quantum efficiency detected there is still quite low, on the order of $\sim 10^{-5}$ photons per electron. To increase the quantum yield and sharpen the spectral selection, in this work we carried out theoretical investigations to explore the feasibility of introducing a PPC structure to further tune the plasmon mode and increase field enhancement. A PPC structure could potentially support both the LSP mode and the resonant cavity mode of a PPC, thus allowing tuning of the coupling between the LSP and cavity mode through geometric modification.

The field enhancement of a cavity is typically described by the Purcell factor defined as $[9,16]$

$$
P=\frac{3 Q \lambda^{3}}{4 \pi^{2} V_{\text {eff }}},
$$


in which $Q$ is the quality-factor, $V_{\text {eff }}$ is the effective volume of the resonant cavity mode and $\lambda$ is the wavelength of light. This formula states that the resonant mode volume $V_{\text {eff }}$ must be minimized to obtain a high $P$ value. By confining the local plasmonic field inside a very small nanocavity, the spontaneous emission rate of molecules inside the nanocavity could be greatly enhanced $[9,17]$. A further advantage of using a PPC structure is to fine tune the cavity mode to match particular molecular vibronic transitions. Sharpened emission could then be achieved through resonant excitation and emission enhancement. When the $Q$-factor of the PPC is sufficiently high, the coupling between the spontaneous emission within the cavity and the lasing mode can be greatly enhanced. This may lead to the generation of superradiance [18] or even plasmonic lasing, often referred to as a spaser [19-22].

In the present study, we studied the optimization of field enhancement and confinement of a two-dimensional (2D) hexagonal PPC with a periodicity of $d$ and dipole sources located around the central vacancy area, by finite-difference time-domain (FDTD) methods. The Fourier spectrum of the time-evolved field is calculated to determine the resonant modes. The relative intensity between the LSP mode and the resonant cavity mode are found to vary with silver $(\mathrm{Ag})$ nanorod radius $r$. Ag was selected as the material for calculation because of its strong surface plasmon effect [23]. Electric field distributions are also calculated for a PPC with a given periodicity (e.g. $d=375 \mathrm{~nm}$ ), which shows an optimized ratio of PPC periodicity to nanorod radius (i.e. $d / r$ ) for the $Q$-factor and field enhancement of the resonant cavity mode. The optimization mechanism is discussed using calculated radiation power spectra through the mode location relative to the photonic stopband and mode energy transfer from the cavity to inter-particle gap plasmons. These results provide insight towards the realization of enhanced spontaneous emission of molecules in nanocavities.

\section{Model and methods}

The system we investigated is a 2D-PPC structure consisting of a hexagonal array of $\mathrm{Ag}$ nanorods with the central rod absent. As shown in Figure 1, $r$ is the radius of the $\mathrm{Ag}$ nanorods and $d$ is the periodicity of the PPC, which was fixed at $375 \mathrm{~nm}$ for all calculations. The small dot at the center represents the magnetic dipole source inserted with orientation along the $z$-direction (i.e. perpendicular to the $2 \mathrm{D}$ plane). M1 is the square monitor surrounding the dipole source(s), which is used to calculate the power injected from the source into the PPC system. M2 is the square monitor surrounding the entire PPC structure, which is used to calculate the power radiating out of the PPC as a function of wavelength. The ratio between the two calculated spectra is the normalized radiation power spectrum, which is used to explain the optimization of the field confinement and the

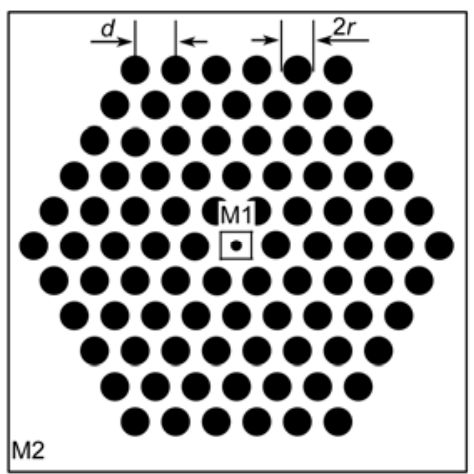

Figure 1 2D-PPC structure consisting of a hexagonal array of Ag nanorods with the central rod absent.

$Q$-factor of the cavity mode. Strong resonant cavity modes can be formed at the defect regime by the Bragg reflection of photons in the periodic structure. The density of photonic states at the cavity can be greatly increased because of the strong localization of light at the nanocavity. This may result in high spontaneous emission rate for the molecule in the cavity.

Two-dimensional FDTD simulations via a rectangular nonuniform mesh were performed using FDTD Solutions software (Lumerical Solutions Inc., Vancouver, Canada) [24]. The grid size used to mesh the PPC structure was set to $d x=3.75 \mathrm{~nm}$ (i.e. $d / 100$ ) and $d y=3.2476 \mathrm{~nm}$ (i.e. $\sqrt{3} d / 200$ ), while that for meshing the region outside the PPC was set to $\sim 10 \mathrm{~nm}$. The effect of the square boundary outside the M2 monitor was also taken into account using a model composed of 12-cell thick perfectly matched layers. The dielectric function of the Ag material was obtained using a fitting routine, to generate a multi-coefficient material model of experimental data [25] over the wavelength range specified by the dipole sources under study. Figure 2 shows a normalized spectrum of the dipole source, which was selected based on our interest in the visible-light region. To map out all the resonant cavity modes, two dipole sources are positioned at two asymmetric points near the central defect, to avoid a null solution for the calculated electric field intensity. The variation in electric field intensity with the time is

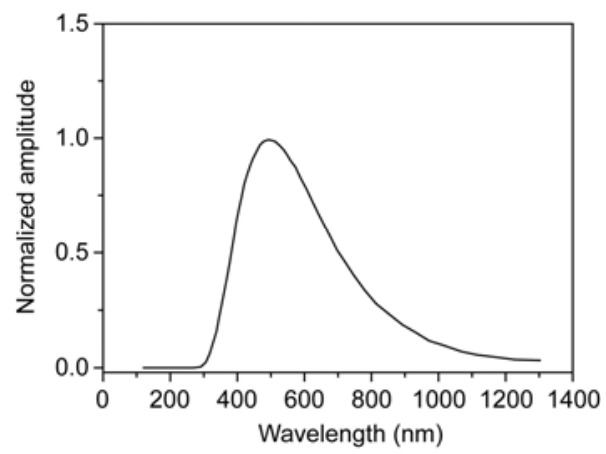

Figure 2 Normalized spectrum of the dipole source. 
calculated at four random points [24]; however, the overall physical picture is independent of point selection. The Fourier spectrum of the time-evolved field is then calculated for different nanorod radii through summation of the Fourier transformation of the time-dependent electric fields and magnetic fields at selected points via the following equation:

$$
\begin{aligned}
& F(\omega)= \\
& \frac{\sum_{n=1}^{N_{1}} \int\left[\left|\int \vec{E}\left(\vec{r}_{i}, t\right) \exp (i \omega t) \mathrm{d} t\right|^{2}+\left(\mu_{0} / \varepsilon_{0}\right)\left|\int \vec{H}\left(\vec{r}_{i}, t\right) \exp (i \omega t) \mathrm{d} t\right|^{2}\right]}{\frac{1}{N_{2}} \sum_{j=1}^{N_{2}}\left|\int \exp (i \omega t) s_{j}(t) \mathrm{d} t\right|^{2}},
\end{aligned}
$$

in which $N_{1}$ refers to the number of points calculated and has a value of 4 in our calculations; $N_{2}$ refers to the number of dipole sources used and equals to 2 for the present calculation; $\vec{E}\left(\vec{r}_{i}, t\right)$ and $\vec{H}\left(\vec{r}_{i}, t\right)$ are the calculated electric and magnetic fields at the $i$ th point, respectively; $s_{j}(t)$ is the source time signal of the $j$ th dipole source in the simulations. The resonant frequency $\omega_{\text {res }}$ and the full width at half maximum of the resonant peak $\Delta \omega$ can be obtained from the calculated spectrum $F(\omega)$. The $Q$-factor can therefore be calculated via $Q=\omega_{\text {res }} / \Delta \omega$. The Purcell factor is calculated via eq. (1) with the effective volume $V_{\text {eff }}$ estimated as the product of the effective area $A_{\text {eff }}$ and the effective height $h_{\text {eff }}$ of the cavity resonant mode. The effective area is calculated by scaling the electric field energy in the PPC structure with the maximum electric field energy density that is confined around the central defect regime of the 2D PPC structure [26]. The effective height is chosen to be $500 \mathrm{~nm}$, which is approximately one wave packet corresponding to the resonant wavelength of the cavity mode.

After obtaining all the resonant cavity modes and their quality and Purcell factors, we set the same dipole exactly at the center. The normalized electric field distribution at the resonant frequency of the cavity mode for different nanorod radii $r$ is then calculated as

$$
\left|\vec{E}\left(\vec{r}, \omega_{\text {res }}\right)\right|^{2}=\left|\frac{\int \exp \left(i \omega_{\text {res }} t\right) \vec{E}(\vec{r}, t) \mathrm{d} t}{\int \exp \left(i \omega_{\text {res }} t\right) s(t) \mathrm{d} t}\right|^{2} .
$$

The power flow through monitors 1 and 2 is calculated as

$$
T_{\mathrm{M} i}(\omega)=\frac{1}{2} \int_{\mathrm{M} i} \operatorname{Re}\left[P\left(\vec{s}_{i}, \omega\right)\right] \cdot \mathrm{d}^{2} \vec{s}_{i},(i=1,2),
$$

where $P(\omega)=\vec{E}(\omega) \times \vec{H}^{*}(\omega)$ is the Poynting vector. Finally, the normalized radiation power is calculated as

$$
R_{\mathrm{nor}}(\omega)=\frac{T_{\mathrm{M} 2}(\omega)}{T_{\mathrm{M} 1}(\omega)}=\frac{\int_{\mathrm{M} 2} \operatorname{Re}\left[P\left(\vec{s}_{2}, \omega\right)\right] \cdot \mathrm{d}^{2} \vec{s}_{2}}{\int_{\mathrm{M} 1} \operatorname{Re}\left[P\left(\vec{s}_{1}, \omega\right)\right] \cdot \mathrm{d}^{2} \vec{s}_{1}} .
$$

\section{Results and discussion}

Figure 3 depicts the change in calculated Fourier spectra of the time-evolved field and resonant modes of PPC for different nanorod radii. Several features are evident: (i) There exist two clear resonant modes in the PPC structure for small $r$ (i.e. $r$ less than $70 \mathrm{~nm}$ ). One is located at $\sim 400 \mathrm{~nm}$ and is attributed to the LSP mode of individual Ag nanorods; the other is at $\sim 500 \mathrm{~nm}$ arising from the resonant cavity mode. The mode assignment can be further justified by the field distribution given in Figure 4. (ii) The relative intensity of the LSP resonance peak with respect to that of the resonant cavity mode changes significantly upon varying the Ag nanorod radii. As $r$ is increased, the resonant cavity mode is strongly enhanced while the LSP signal becomes less and less discernable. Such $r$-dependency for resonant modes of the PPC can be rationalized as follows: As $r$ is increased, the LSP resonance of the single Ag nanorod becomes weaker because of its larger radius of curvature at the surface [1]. Meanwhile, the distance between neighboring Ag nanorods in the PPC structure becomes smaller and as a

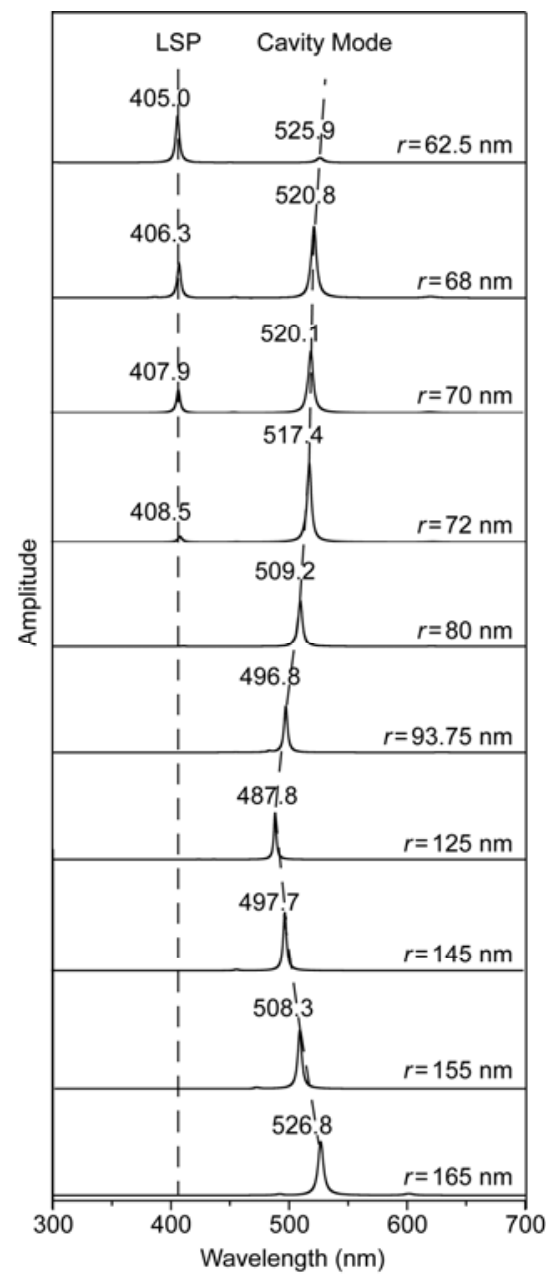

Figure 3 Fourier spectra of the time-evolved field for the resonant modes of the PPC, as a function of nanorod radii $r$. 
result, the electromagnetic coupling between $\mathrm{Ag}$ nanorods becomes stronger, leading to the formation of a stronger resonant cavity mode. The observed rapid diminishing of the relative intensity of the LSP signal with respect to that of the resonant cavity mode in Figure 3 is the combined result of the above two factors. (iii) Of particular interest is the spectral tuning feature for the cavity resonant frequency, and the occurrence of a critical condition for achieving the maximum energy of this resonant cavity mode. While the peak position of the LSP mode appears to be relatively constant, the resonant frequency of the cavity mode can be tuned over a relatively wide energy range by changing the nanorod radii, e.g. from $\sim 526 \mathrm{~nm}$ at $r=62.5 \mathrm{~nm}$ to $\sim 488 \mathrm{~nm}$ at $r=125 \mathrm{~nm}$. In the present configuration setup, $125 \mathrm{~nm}$ appears to be a critical nanorod radius, represented by $r_{\mathrm{c}}=$ $d / 3$, for the PPC structure to have a maximum resonant cavity energy. The gap separation between neighboring nanorods also equals $125 \mathrm{~nm}$ under this critical condition. Along with the increase in the nanorod radius, the resonant peak of the cavity mode is blue-shifted for $r<r_{\mathrm{c}}$ and red-shifted for $r>r_{\mathrm{c}}$. These observations suggest that the resonant cavity mode can be tuned to match with particular optical transitions of quantum emitters like molecules, by changing the
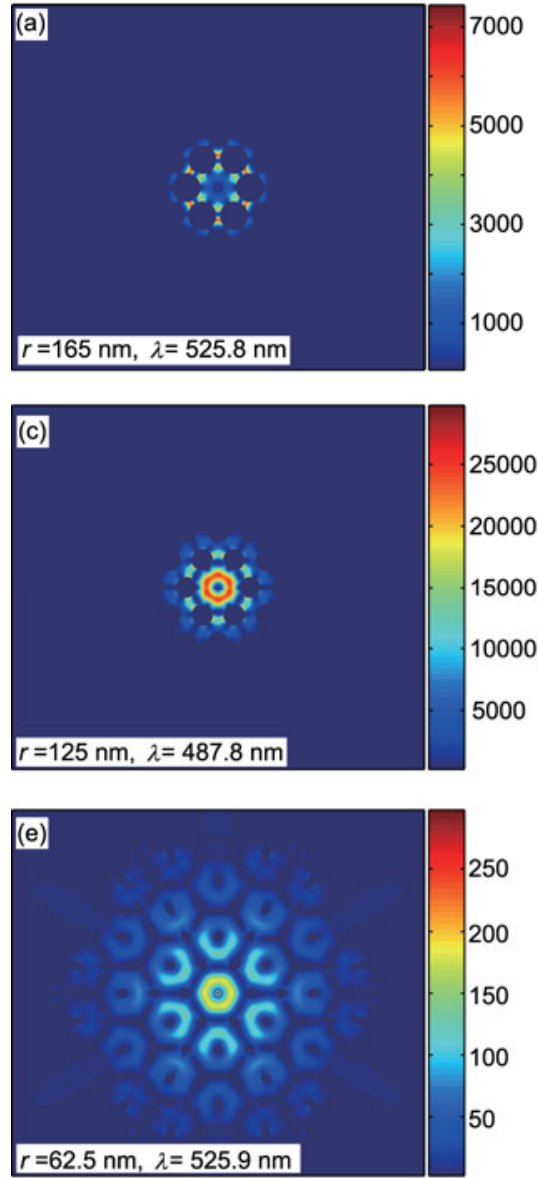

geometric parameters of the PPC so that strong resonant excitation can be obtained.

To examine how the critical condition affects the Purcell enhancement of the cavity, we show in Figure 4 the field distributions at the resonant wavelength $(\sim 500 \mathrm{~nm})$ of the cavity mode for different $r$. The field distribution at the LSP resonant wavelength $(\sim 405 \mathrm{~nm})$ for $r=62.5 \mathrm{~nm}$ is also shown for comparison, to illustrate the differences in field enhancement and spatial distribution of resonant modes.

There are two prominent features in Figure 4. The first and perhaps most important is what happens at the critical nanorod radius condition. When $r_{\mathrm{c}}=d / 3=125 \mathrm{~nm}$, the resonant cavity mode exhibits the strongest field enhancement, up to 29000 for the field intensity $\left(|E|^{2}\right)$. Under this critical condition, the resonant cavity mode also has the strongest field confinement with an effective mode area down to $\sim 0.255 \mu \mathrm{m}^{2}$. This critical situation is also reflected in the $Q$-factor and the Purcell factor $P$. Plots of $Q$-factors and Purcell factors versus the radius $r$ in Figure 5 indicate that both factors reach a maximum value at $r_{\mathrm{c}}=125 \mathrm{~nm}, \sim 200$ for the $Q$-factor and $\sim 15$ for the Purcell factor. These results suggest that at the critical nanorod radius condition, the resonant cavity mode offers the strongest field confinement
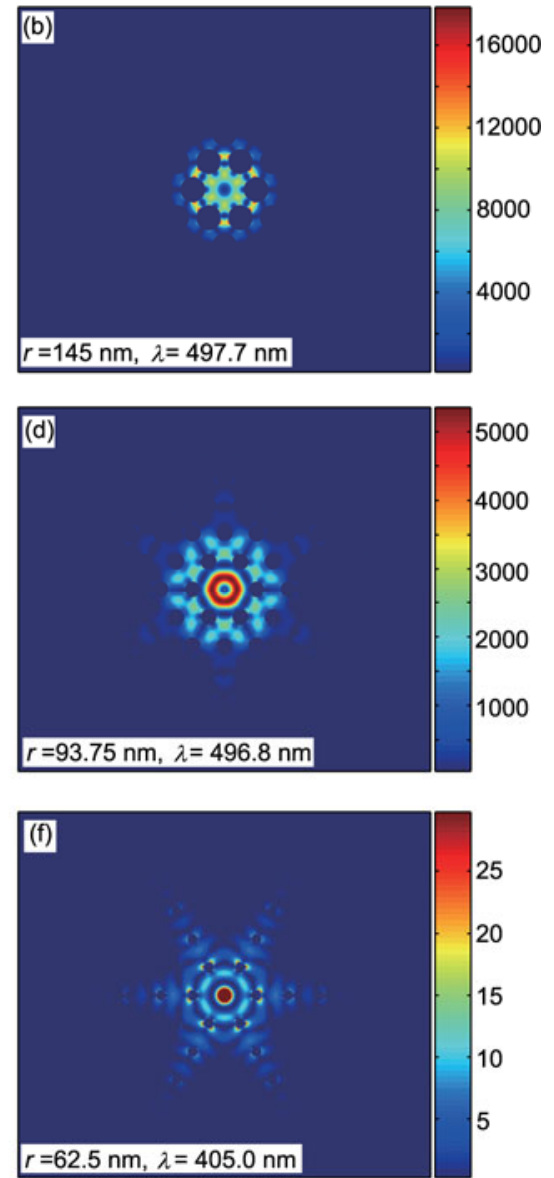

Figure 4 (a)-(e) Field distributions $\left(|E|^{2}\right)$ at the resonant wavelength of the cavity mode for varying $r$; (f) field distribution of the LSP resonant mode of individual $\mathrm{Ag}$ nanorods for $r=62.5 \mathrm{~nm}$. 


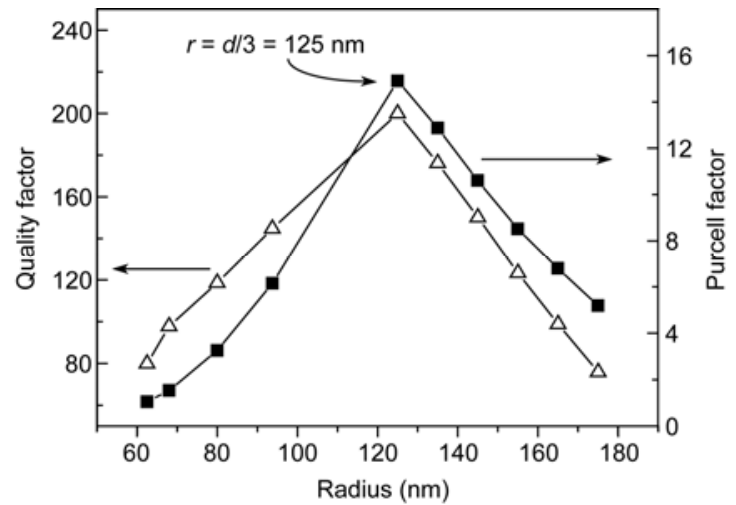

Figure 5 Calculated $Q$-factors (open triangles) and Purcell factors (solid rectangles) of the resonant cavity mode versus $r$. The maximum value occurs at $r=r_{\mathrm{c}}=d / 3$.

and enhancement, as well as the best energy storage capability and probably also the longest photon lifetime [27]. These features may create favorable conditions for the resonant excitation of molecules in the cavity and lead to enhanced spontaneous emission rates.

The second feature is related to the changing trend of field distribution as a function of nanorod radii, both on the intensity and spatial location of resonant cavity modes. For $r>r_{\mathrm{c}}$, the maximum field intensity of the resonant cavity mode is still relatively strong. However, it is localized at the inter-particle gap between the neighboring $\mathrm{Ag}$ nanorods, because of the stronger inter-particle plasmon interaction. By contrast, the local electric field for the resonant cavity mode around the central defect regime is decreased significantly, and this tendency is also observed for $r<r_{\mathrm{c}}$ except decreasing at a faster speed with a Purcell enhancement down to 1 at $r=62.5 \mathrm{~nm}$ (Figures 4 and 5). In this case, the electric field of the resonant cavity mode is no longer confined to the small space around the dipole source, and thus light cannot be squeezed into the central regime.

Figure 4 also indicates that the resonant cavity mode at $\sim 500 \mathrm{~nm}$ in Figure 3 is primarily localized around the central defect regime, in particular for the maximum Purcell enhancement at the critical nanorod radius. For the LSP mode at $\sim 400 \mathrm{~nm}$, it is predominantly localized around the nanorod itself, as illustrated in Figure 4(f), through the dipolar pattern of the field distribution of individual Ag nanorods. The field enhancement of the LSP resonant mode is much weaker than that of the resonant cavity mode (see Figure 4(c) and (f)). This observation suggests that the resonant cavity mode of a PPC structure may increase the efficiency of many optical processes to a large extent, with the enhancement effect much greater than what could be achieved by the LSP enhancement alone.

To understand the optimization mechanism of the field enhancement and the $Q$-factor of the cavity mode, we calculated the normalized radiated power for different $r$. As shown in Figure 6(a), for $r<r_{\mathrm{c}}$, no photonic stopband is formed in the range of visible light for the normalized radi- ation power spectra. Shallow dips in the spectra at the resonant wavelength (marked by arrows) arise from the field confinement property of the resonant cavity mode. Because the resonance wavelength of the cavity mode is outside the photonic stopband, the energy of the cavity mode can easily escape from the cavity and the light confinement property of the cavity mode is poor. As a result, either the $Q$-factor or the Purcell enhancement is small. Nevertheless, as $r$ is increased, the dip becomes deeper, which implies a better field confinement. In particular, when $r$ is increased to the critical radius $r_{\mathrm{c}}$ (i.e. $125 \mathrm{~nm}$ ), a photonic stopband is opened up in the visible range with one band edge located at $\sim 500 \mathrm{~nm}$ (Figure 6(b)), and equally importantly, the resonant wavelength of the cavity mode, $488 \mathrm{~nm}$ shown in Figure 3, also falls inside the photonic stopband. Consequently, the confined light of the resonant cavity mode cannot radiate out from the nanocavity, which results in a highly confined field and therefore a strong local field enhancement in the cavity. This efficient energy confinement also leads to a high $Q$-factor and Purcell factor. However, as $r$ is further increased above $r_{\mathrm{c}}$ (e.g. to 145 and $165 \mathrm{~nm}$ ), the separation between neighboring $\mathrm{Ag}$ nanorods decreases to dozens of nanometers and a strong localized inter-particle plasmon mode forms instead. The field is more confined at the gaps between the Ag nanorods in the first central ring than those in the central cavity regime (Figure 4(a) and (b)). The coupling between the cavity mode and inter-particle plasmons will lead to an energy transfer from the former to the later. Therefore, although the resonant wavelength of the
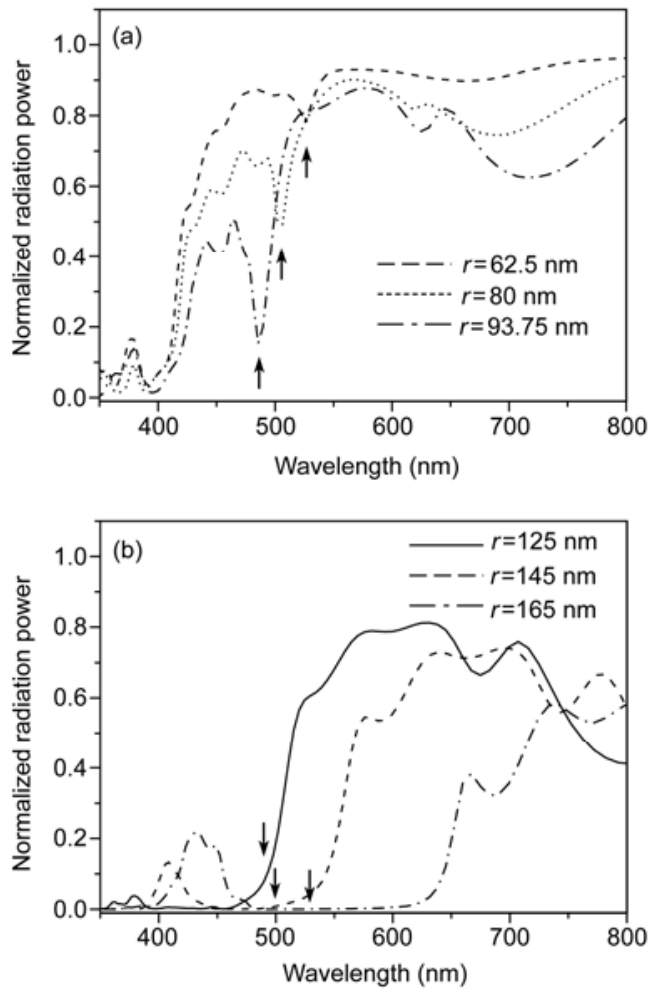

Figure 6 Normalized radiation power spectra versus $r$. (a) $r<r_{\mathrm{c}}$; (b) $r \geqslant r_{\mathrm{c}}$. 
cavity mode for $r>r_{\mathrm{c}}$ moves into the middle region of the opened photonic stopband, the local field intensity inside the central nanocavity is still reduced because of energy transfer to inter-particle plasmons. As a result, both the $Q$-factor and the Purcell factor are decreased as $r$ is further increased above $r_{\mathrm{c}}$, yielding the observed optimization situation at the critical nanorod radius.

The critical condition of $r_{\mathrm{c}}=d / 3$ for the field enhancement appears universal for other periodicities, provided that the LSP mode and cavity resonant mode are well separated. This is confirmed by the calculated results for $d$ values of $330,450,510$ and $570 \mathrm{~nm}$. The physical origin behind the $r_{\mathrm{c}}=d / 3$ critical condition is currently unclear and requires further investigation, but it might be related to the Bragg reflection. It should be noted that the normalized radiation power spectra in Figure 6 also suggest the possibility of tuning the magnitude and band-edge energy of opened photonic stopbands through changing nanorod radii.

\section{Conclusions}

We theoretically investigated the optimization of electric field enhancement of the resonant cavity mode, by varying metallic nanorod radii in a 2D-PPC structure. An optimized condition for the $Q$-factor and the Purcell enhancement of the cavity was found for a given PPC periodicity with the critical nanorod radius $r_{\mathrm{c}}=d / 3$. At this point the resonant cavity mode has the strongest field enhancement, best field confinement and largest $Q$-factor. This enhancement optimization is attributed to competition between the blocking of cavity confined light to radiate out when the cavity resonant frequency falls inside the opened photonic stopband as $r$ reaches $r_{\mathrm{c}}$, and the transfer of cavity mode energy to interparticle plasmons when $r$ is further increased. Under the critical nanorod radii condition, light can be squeezed into the smallest space with the strongest field enhancement and best quality factor of a cavity. This condition offers the highest density of photonic states in the resonant nanocavity. Our calculation results also indicate a possibility not only to tune the maximum field enhancement, but also to tune the energy of resonant cavity mode and the position of photonic stopband through the nanorod radii so that strong resonant excitation can be realized. These results may provide new routes to the resonant enhancement of spontaneous emission rates of quantum emitters in nanocavities, and lead to an improvement in the quality of PPC plasmonic nanolasers.

This work was supported by the National Basic Research Program of China (2006CB922003, 2011CB921402), Chinese Academy of Sciences (KJCX2.YW.H06) and National Natural Science Foundation of China (91021004, 10574117, 10974186).
1 Wang Z L. A review on research progress in surface plasmons (in Chinese). Prog Phys, 2009, 29: 287-324

2 Hillenbrand R, Taubner T, Keilmann F. Phonon-enhanced lightmatter interaction at the nanometre scale. Nature, 2002, 418: 159-162

$3 \mathrm{Xu} \mathrm{H} \mathrm{X,} \mathrm{Bjerneld} \mathrm{E} \mathrm{J,} \mathrm{Käll} \mathrm{M,} \mathrm{et} \mathrm{al.} \mathrm{Spectroscopy} \mathrm{of} \mathrm{single} \mathrm{hemo-}$ globin molecules by surface enhanced Raman scattering. Phys Rev Lett, 1999, 83: 4357-4360

4 Nie S, Emory S R. Probing single molecules and single nanoparticles by surface-enhanced Raman scattering. Science, 1997, 275: 1102-1106

5 Kneipp K, Wang Y, Kneipp H, et al. Single molecule detection using surface-enhanced Raman scattering (SERS). Phys Rev Lett, 1997, 78: $1667-1670$

6 Anger P, Bharadwaj P, Novotny L. Enhancement and quenching of single-molecule fluorescence. Phys Rev Lett, 2006, 96: 113002

7 Lakowicz J R. Radiative decay engineering 5: Metal-enhanced fluorescence and plasmon emission. Anal Biochem, 2005, 337: 171-194

8 Wei H, Hao F, Huang Y Z, et al. Polarization dependence of surface-enhanced Raman scattering in gold nanoparticle-nanowire systems. Nano Lett, 2008, 8: 2497-2502

9 Gelfand R M, Bruderer L, Mohseni H. Theoretical design for a plasmon-polariton photonic crystal. In: 21st Annual Meeting of the IEEE Lasers and Electro-Optics Society, 2008, 143-144

10 Lee S J, Guan Z Q, Xu H X, et al. Surface-enhanced Raman spectroscopy and nanogeometry: The plasmonic origin of SERS. J Phys Chem C, 2007, 111: 17985-17988

11 Li J Q, Zhang Y, Mei T, et al. Surface plasmon laser based on metal cavity array with two different modes. Opt Exp, 2010, 18: 2362623632

12 Wang B, Wang G. Plasmon Bragg reflectors and nanocavities on flat metallic surfaces. Appl Phys Lett, 2005, 87: 013107

13 Liu J L, Lin J, Zhao H F, et al. Numerical analysis of surface plasmon nanocavities formed in thickness-modulated metal insulator metal waveguides. Chin Phys B, 2010, 19: 054201

14 Dong Z C, Zhang X L, Gao H Y, et al. Generation of molecular hot electroluminescence by resonant nanocavity plasmons. Nat Photon, 2010, 4: 50-54

15 Dong Z C, Guo X L, Trifonov A S, et al. Vibrationally resolved fluorescence from organic molecules near metal surfaces in a scanning tunneling microscope. Phys Rev Lett, 2004, 92: 086801

16 Purcell E M. Spontaneous emission probabilities at radio frequencies. Phys Rev, 1946, 69: 681

17 Kleppner D. Inhibited spontaneous emission. Phys Rev Lett, 1981, 47: 233-236

18 Pitanti A, Bettotti P, Sarchi D, et al. Purcell factor and superradiance in Si-patterned waveguides. Opt Lett, 2010, 35: 3384-3386

19 Okamoto T, H'Dhili F, Kawata S. Towards plasmonic band gap laser. Appl Phys Lett, 2004, 85: 3968-3970

20 Zheludev N I, Prosvirnin S L, Papasimakis N, et al. Lasing spaser. Nat Photon, 2008, 2: 351-354

21 Bergman D J, Stockman M I. Surface plasmon amplification by stimulated emission of radiation: Quantum generation of coherent surface plasmons in nanosystems. Phys Rev Lett, 2003, 90: 027402

22 Noginov M A, Zhu G, Belgrave A M, et al. Demonstration of a spaser-based nanolaser. Nature, 2009, 460: 1110-1112

23 Johansson P. Light emission from a scanning tunneling microscope: Fully retarded calculation. Phys Rev B, 1998, 58: 10823-10834

24 FDTD Solutions Getting Started. Release 7.5. Lumerical Solutions, Inc., 2011

25 Johnson P B, Christy R W. Optical constants of the noble metals. Phys Rev B, 1972, 6: 4370-4379

26 Painter O, Lee R K, Scherer A, et al. Two-dimensional photonic band-gap defect mode laser. Science, 1999, 284: 1819-1821

27 Takahashi Y, Hagino $\mathrm{H}$, Tanaka $\mathrm{Y}$, et al. High- $Q$ nanocavity with a 2-ns photon lifetime. Opt Exp, 2007, 15: 17206-17213

Open Access This article is distributed under the terms of the Creative Commons Attribution License which permits any use, distribution, and reproduction in any medium, provided the original author(s) and source are credited. 